\title{
Assessment and Tracking Electric Vehicle Battery Degradation Cost using Blockchain
}

\author{
Shashank Narayana Gowda \\ Smart Grid Energy Research Center \\ University of California, Los Angeles \\ Los Angeles, USA \\ shashank07@ucla.edu
}

\author{
Hamidreza Nazaripouya \\ School of Electrical and Computer Engineering \\ Oklahoma State University \\ Stillwater, USA \\ hanazar@okstate.edu
}

\author{
Basem A. Eraqi \\ Physical Science and Engineering Division \\ King Abdullah University of Science and Technology (KAUST) \\ Thuwal, Saudi Arabia \\ basem.eraqi@kaust.edu.sa \\ Rajit Gadh \\ Smart Grid Energy Research Center \\ University of California, Los Angeles \\ Los Angeles, USA \\ rgadh@seas.ucla.edu
}

\begin{abstract}
This paper proposes a blockchain-based method for assessment and tracking of electric vehicle battery degradation costs. Vehicle-to-Grid (V2G) technology allows the bidirectional flow of electric power between the electric vehicle ( $\mathrm{EV})$ and the electric grid. However, for making optimal charging/discharging decisions, it is essential to precisely evaluate the battery degradation. In the proposed method, the initial degradation cost is estimated based on the present status of the EV battery in terms of range and age. Following this, a degradation cost of the battery is obtained from battery specifications and continuously tracking the variables that affect battery energy capacity, which will determine the economic loss to EV users for participating in V2G programs. A Mixed Integer Linear Program to minimize cost includes this degradation cost in its objective function to make the optimal decision for an EV's interaction with the grid. At the end of each 24-hour cycle, the battery degradation cost is updated based on the charging/discharging transactions performed during the cycle and the temperature conditions. These transactions and battery degradation costs are stored in a consortium blockchain that is shared among the relevant actors.
\end{abstract}

Index Terms-Electric vehicles, V2G, battery degradation, blockchain.

\section{INTRODUCTION}

Vehicle-to-Grid (V2G) is a promising technology that utilizes Electric Vehicle (EV) batteries during the prolonged periods the vehicles are not used for transportation. V2G enables the flow of power from an EV's battery back to the grid. Utilizing V2G for grid services provides the power systems with numerous benefits, such as increased renewable energy penetration, better peak shaving, frequency and voltage regulation, and backup power supply. One of the major challenges facing V2G is battery degradation caused by frequent charging/discharging. The economic cost of this degradation makes it difficult to conclude the cost-effectiveness of utilizing V2G. Depending on multiple variables, including degradation cost, the price of electricity, the revenue from grid services (i.e. frequency regulation, spinning reserves, etc.,) and availability of renewable energy, the grid operator needs to decide whether or not to utilize V2G.

Multiple research articles have ignored battery degradation while studying the feasibility of V2G [1], [2]. H. Farzin et al. presented a methodology for calculating battery degradation due to V2G in terms of depreciation cost so that it could be incorporated in the objective function for charging/discharging optimization [3]. This method is used in more recent research articles [4]. The drawbacks include (i) iterative procedure to compute battery degradation cost, (ii) ignoring the calendar aging, and (iii) ignoring the effects of charge/discharge rates. An iterative procedure is used to estimate the battery degradation cost and assumes this parameter is constant for the lifetime of the EV. However, the degradation cost is not constant and depends on the number of charging/discharging cycles and depth of discharge. Each future cycle and varying Depth of Discharge (DOD) will affect the degradation cost [3]. Also, as the number of vehicles willing to take part in V2G increases, the optimization programs will take a larger amount of time to converge. Calendar aging is one of the most important variables in deciding for or against V2G. If the age of the battery is not tracked, the degradation cost values will not accurately portray the state of the battery. The charge/discharge rates also affect the battery life of an EV, fast charging degrades the battery more rapidly [4]. This paper aims to provide the conceptual, mathematical and technological framework for assessing the economic viability of using V2G capability in electric vehicles. The battery degradation cost will be computed from battery specifications, and engineering economics principles. This degradation cost will be included in a Mixed Integer Linear Program for optimal charging/discharging decisions. Following a 24-hour cycle of transactions, the battery degradation cost will be updated at the end of the cycle based on charging/discharging parameters and battery temperature conditions to include the effects of calendar aging. All transactions and degradation costs are 


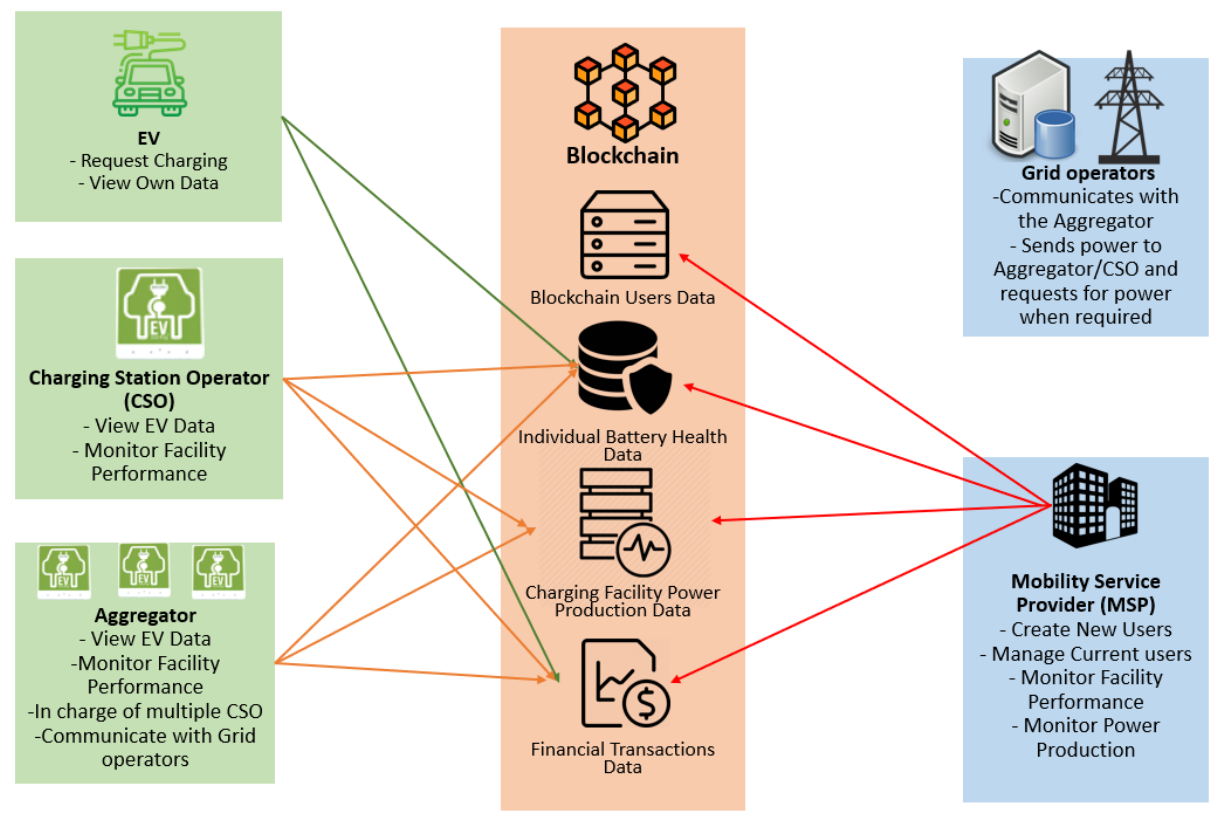

Fig. 1. A network fabric diagram showing the different data access authorization for all actors in the blockchain private network.

logged into a consortium blockchain that relevant actors have access to.

\section{BLOCKCHAIN}

A blockchain is a distributed ledger that links blocks (records of data) using cryptography. Blockchain was introduced in Bitcoin [5] which is a digital cryptocurrency that is operated by a decentralized authority. Blockchains and rely on consensus mechanisms to verify the correctness of transactions that are written onto it. Blockchains are generally classified as public, consortium, and private blockchains. Public blockchains can lead to a lack of privacy, higher costs, and slower processing while private blockchains place restrictions on who can participate. A consortium blockchain requires a group of equally powerful parties to validate the transactions instead of a single entity or any participant on the blockchain. The blockchain visibility is limited to validators and authorized individuals. Blockchains have been used in the energy sector for energy trading [6], battery swapping [7], internet of things, [8] and so on. The consortium blockchain is the most appropriate for tracking the lifetime of the battery in the $\mathrm{EV}$ because the transactions have to be validated by multiple involved actors who benefit from the EV charging/discharging.

The blockchain network is defined on the collection of EV users, EV Charging Station Operators (CSOs), Aggregators, and EV Mobility Service Providers (MSP). Each actor in the network has a unique set of authorizations and restrictions which will allow the blockchain to achieve its intended goals. The EV CSOs are responsible for charging EVs. The Aggregator communicates with and controls the charging stations in several CSO facilities. Since grid services mostly require power in the order of hundreds of $\mathrm{kW}$ or a few MW, the Aggregator would apply for the required service in the market and then fulfill the promised application through the aggregation of EVs. The EV MSP connects the various EVs to EV CSOs and Aggregators at different locations. It ensures that the EV can charge at multiple CSOs and participate in V2G with multiple Aggregators. All the actors involved would like to maximize their profits at the cost of the other actors if unregulated. Since the consortium blockchain requires transaction validation from all the relevant actors, it is the most appropriate for logging and validating transactions, and using these transactions for tracking EV battery life.

The blockchain will track the charging/discharging power and operating temperature data for each EV which will be used to generate the EV's battery degradation cost. The degradation cost is used by the aggregator to run optimization programs and decide if an EV should participate in V2G applications. EV owners will be compensated for allowing their EVs to participate in $\mathrm{V} 2 \mathrm{G}$ applications which currently include demand response [9], frequency regulation [10], renewable energy compensation [11] and possibly even infrastructure deferral and transmission congestion [12]. The profits from V2G will be distributed among the actors. Along with the power transaction history, the distribution of money among the actors for each 24-hour cycle is stored.

The EV user has READ access allowing the user to view the charging/discharging data log of their respective vehicle along with the income earned for $\mathrm{V} 2 \mathrm{G}$ and charging expenses. They cannot access the data of other EVs.

The EV CSO and the Aggregator have READ access, allowing them to read the charging/discharging transactions for each EV and the EV battery degradation cost. The Aggregator will utilize the battery degradation cost data to decide if the EV can participate in a V2G application and which 
set of vehicles will be involved in the $\mathrm{V} 2 \mathrm{G}$ activity. They also have WRITE access to write power transactions, EV battery degradation cost, and monetary transactions on the blockchain. The Aggregator directly communicates with the Distribution Network Operator (DNO) or the Transmission System Operator (TSO) depending on the V2G application promised. Figure 1 highlights the information that each actor has access to.

In this paper, Hyperledger Fabric, an open-source blockchain development environment is used to design the blockchain and develop the code. Hyperledger composer is used for initial testing. Javascript is used to implement the network transaction logic, which consists of defining the different types of transactions and involved actors. After designing the network based on the design parameters mentioned above, each user is tested against their intended privileges and restrictions. The ledger is also tested for the integrity and completeness of the data. One of the benefits of the Hyperledger blockchain is that it requires permission for a participant to join and make transactions while increasing security against cyber-attacks.

\section{BATtERy DegRAdation Model}

EVs primarily utilize Li-ion batteries which are a complex technology. In Li-ion batteries, capacity fade may be caused due to multiple reasons like the number of cycles, temperature, and operating conditions. EV's Battery Managements Systems (BMS) tracks the state variables of the battery like voltages, currents, and temperature. These are important for estimating the battery's State of Charge (SOC) and State of Health (SOH). Chemical and mechanical changes that occur at different system operations affect the SOH of the battery. The three main types of models that are used to estimate $\mathrm{SOH}$ are datadriven models, physics-based models and equivalent circuit models [13]. The BMS uses simple equivalent circuit models to estimate the SOC and SOH since they are computationally efficient in embedded systems. Physics-based models are accurate but computationally slower and data-driven models require some historical battery operation data.

In this paper, we will be using the model developed in [14], which has been used for comparing battery degradation in normal operation versus V2G [15]. In general, the collected data using the blockchain and the proposed procedures in this paper are compatible with any battery model.

The current State of Health ( $\mathrm{SOH})$ of the EV battery can be estimated based on the range and age of the EV. The initial range and age of the EV will be one-time inputs provided by the user. These metrics will initialize the blockchain process that tracks the battery degradation cost to the end of its life.

The calendar loss $Q_{c a l}$ is a function of temperature $(T)$ and time $(t)$, it can be estimated using equation (1) [14] where $\mu$ is the pre-exponential factor, which is 14876 day $^{-1 / 2} . E_{a}$ is the activation energy which equals $24.5 \mathrm{~kJ} / \mathrm{mol}$ and $R$ is the gas constant, which is $8.314 \mathrm{~J} / \mathrm{mol} \mathrm{K} . T$ is the absolute temperature in Kelvin and $t$ is the time in days.

$$
Q_{c a l}=\mu e^{\frac{-E_{a}}{R T}} t^{0.5}
$$

The cycle loss is a function of temperature $(T)$ and charge/discharge rate and current throughput [14]. It is estimated based on equations (2), (3), and (4). Equation (2) estimates the cycle loss while equations (3) and (4) find the pre-exponential factor $B_{1}$ and the exponential factor $B_{2}$ respectively, both of which are functions of temperature. The values of $a, b, c, d$ and $e$ are obtained from the model developed in [14]. The $C_{\text {rate }}$ is the charge/discharge factor expressed as $\mathrm{C}$-rate and the $A_{h}$ is the cumulative current throughput in $\mathrm{Ah}$.

$$
\begin{gathered}
Q_{\text {cycle }}=B_{1} e^{B_{2} C_{\text {rate }}} A_{h} \\
B_{1}=a T^{2}+b T+c \\
B_{2}=d T+e
\end{gathered}
$$

\section{OPTIMIZATION FRAMEWORK}

A MILP (Mixed Integer Linear Program) is used to schedule the charging/discharging behavior of EVs with an objective to minimize the cost of charging and degradation of $20 \mathrm{EVs}$ in an EV CSO facility. Python and Gurobipy are chosen as main programming/simulation environments. A degradation cost that captures EV battery cycle degradation as a $\$ / \mathrm{kWh}$ value is introduced in the objective. The optimization is run for a 3 year period with discrete time intervals of 1 hour. Equations (1) and (2) are discretized to follow the same hourly intervals, and rewritten as equations (5) and (6). Equations (5) and (6) represent the calendar aging and cycle aging for time $\hat{t}$. The cumulative cycle and calendar losses are the sum of all the losses at the various time intervals values as shown in equation (7).

$$
\begin{gathered}
\Delta Q_{\text {cal }}(\hat{t})=0.5 \mu e^{\frac{-E_{a}}{R T}} \hat{t}^{-0.5} \Delta t \\
\Delta Q_{\text {cycle }}(\hat{t})=B_{1} e^{B_{2} C_{\text {rate }}} I_{\text {rate }} \Delta t \\
Q_{\text {net }}(t)=\Sigma_{\hat{t}=0}^{t}\left(\Delta Q_{\text {cal }}(\hat{t})+\Delta Q_{\text {cycle }}(\hat{t})\right)
\end{gathered}
$$

Thermal models can be used to supplement battery degradation models. However, this model assumes the battery temperature to be ambient temperature. The temperature data for each day in the model is assumed to be the average temperature in Los Angeles for that day for the years 2017-2019.

The optimization objective and constraints are listed in equations (8) to (18). The optimal value for the 24-hour period is used to calculate the total cycle loss for that day based on equation (6) and then the degradation cost based on equation (19). Assume a 24-hour cycle is divided into $\mathrm{T}$ time intervals and the number of EVs willing to provide V2G services is $N_{e v}$. For day $i$, EV $j$, and time interval $k$, the MILP objective is equation (8). The objective minimizes the charging cost and degradation cost. For the day $i, P_{c h, k, j}$ and $P_{d c h, k, j}$ represent the charging power and discharging power in $\mathrm{kW}$ for $\mathrm{EV} j$ at time interval $k . C_{c h, k, j}$ and $C_{d c h, k, j}$ are the cost of charging and profits from discharging respectively in $\$ / \mathrm{kWh}$ for EV 
$j$ at time interval $k$ on the day $i$. The charging rates are based on LADWP R1B 2019 rate structure. Without loss of generality, we assume that the EV will be participating in energy arbitrage, but the revenue from discharging can be set to the value representing other applications at the various time intervals. $\eta_{c h}$ and $\eta_{d c h}$ are the charging and discharging efficiencies which are set at $90 \% . w_{i, j}$ is the degradation cost with units of $\$ / \mathrm{kWh}$.

$$
\begin{array}{r}
\min \sum_{j=1}^{N e v} \sum_{k=1}^{T}\left[\left(P_{c h, k, j} C_{c h, k, j}-P_{d c h, k, j} C_{d c h, k, j}\right)\right. \\
\left.+\left(P_{c h, k, j} \eta_{c h}+P_{d c h, k, j} / \eta_{d c h}\right) w_{i, j}\right]
\end{array}
$$

The constraints are represented in equations (9) to (18). Equation (9) states that the energy in $\mathrm{EV} j$ at time $k$ is the sum of the energy in $\mathrm{EV} j$ at time $k-1$ and the power transferred to or from the EV for the duration $\Delta T$. Equation (10) sets limitations on the maximum and minimum energy stored in the battery of EV $j$. The energy in the EV $j$ at any time interval $k$ is assumed to lie between $10 \%$ and $90 \%$ of the EV battery's nominal capacity. Equation (11) and (12) set limitations on the charging and discharging power of the $\mathrm{EV}$ when it is plugged into an EV charger with charging and discharging power limitations of $P_{c h, \max }$ and $P_{d c h, \max }$ respectively. $\delta_{c h, k, j}$ and $\delta_{d c h, k, j}$ are binary decision variables as shown in equations (13) and (14). The sum of $\delta_{c h, k, j}$ and $\delta_{d c h, k, j}$ is always less than or equal to 1 as shown in equation (15). This indicates that the EV can have one of three statuses for a time interval $k$ when plugged in - charging, discharging, or idle. Equation (16) defines the energy in the EV $j$ on arrival on the day $i$ and equations (17) states that the energy in the EV $j$ at departure on the day $i$ should be at minimum the desired charge expected by the EV user. Equation (18) sets limits on the total power into and out of the EV CSO infrastructure. Constraints (9)-(18) hold when the EV is plugged into the charger. When the EV is unplugged, $\delta_{c h, k, j}$ and $\delta_{d c h, k, j}$ are set to zero.

$$
\begin{gathered}
E_{k, j}=E_{k-1, j}+\left(P_{c h, k, j} \eta_{c h}-P_{d c h, k, j} / \eta_{d c h}\right) \Delta T \\
E_{\text {min }, j} \leq E_{k, j} \leq E_{\max , j} \\
0 \leq P_{c h, k, j} \leq \delta_{c h, k, j} P_{c h, \max } \\
0 \leq P_{d c h, k, j} \leq \delta_{d c h, k, j} P_{d c h, \text { max }} \\
\delta_{c h, k, j} \in 0,1 \\
\delta_{d c h, k, j} \in 0,1 \\
\delta_{c h, k, j}+\delta_{d c h, k, j} \leq 1 \\
E_{a r r, j}=E_{i n i, j} \\
E_{d e p, j} \geq E_{d e s, j} \\
-\sum_{\text {inf }} \leq \sum_{j=1}^{N e v} P_{c h}-\sum_{j=1}^{N e v} P_{d c h} \leq P_{\text {inf }}
\end{gathered}
$$

At the end of the day, based on data including charging/discharging schedules and the capacity losses, a new estimate will be made for battery degradation costw for the following day. Equation (19) estimates the degradation cost $w_{i, j}$ for day $i$ and $\mathrm{EV} j$ with units of $\$ / \mathrm{kWh}$, based on the cumulative calendar and cycle losses. $\triangle B P$ is the difference in the original battery price and the salvage price (assumed to be $20 \%$ of the original battery price) in $\$$. The battery's end of life is assumed to occur when the battery loses $30 \%$ of its initial capacity. $E_{c a p, j}$ is the EV's original battery capacity in $\mathrm{kWh}$ and $Q_{t o t, i-1, j}$ is the cumulative capacity losses for EV $j$ from day 0 to day $i-1$. Each day's EV energy transactions, monetary transactions, and End of Day (EoD) battery degradation cost will be added to and maintained in the consortium blockchain.

$$
w_{i, j}=\frac{\Delta B P}{30\left(100-Q_{t o t, i-1, j}\right) E_{c a p, j}}
$$

The optimization is run for twenty new Nissan Leaf EVs for a three-year period to generate daily charging costs, estimate battery degradation costs, transfer the information to the blockchain and read the degradation cost information for the next day's optimization program. The nominal battery capacity of all the EVs is $40 \mathrm{kWh}$. The EV is charged at the workplace between 7 am and $7 \mathrm{pm}$. The EV arrives with $40 \%$ SOC and requires a minimum of $60 \%$ SOC at departure. The optimization is run for two different degradation costs - (i) constant degradation cost at $0.04 \$ / \mathrm{kWh}$ and (ii) dynamic degradation cost as obtained from equation (19) for a maximum charging/discharging rate of $6.6 \mathrm{~kW}$. The $\mathrm{V} 2 \mathrm{G}$ application considered here is energy arbitrage.

\section{Results}

The results for constant and dynamic degradation costs over the three-year period are summarized in Table 1. The revenue from participating in energy arbitrage is $\$ 2447.6$ for the case with dynamic degradation cost which is $31.7 \%$ higher than the $\$ 1880.9$ in the constant degradation cost case. The total energy supplied by V2G is $381.6 \mathrm{MWh}$ for the case with dynamic degradation cost which is only $2.5 \%$ higher than the 372.3 MWh in the constant degradation cost case. The V2G energy per EV is in the range of 7.23 to $27.37 \mathrm{MWh}$ in case of the constant degradation cost while the range is between 19.07 and 19.09 MWh for the dynamic degradation cost. Effectively all the EVs in the dynamic degradation cost are contributing equally because they all started with the same initial battery energy capacity. Figure 2 and Table 1 highlight the difference in the battery energy capacity of the EVs at the end of the three-year period. The dynamic degradation cost prioritizes EVs with lower battery degradation for $\mathrm{V} 2 \mathrm{G}$ in each 24-hour cycle resulting in all the EVs having similar remaining battery capacity around $70 \%$ while the EVs using the constant degradation cost have remaining battery capacity in the range 67.8 to $75.6 \%$.

While the EVs can participate in various V2G applications to support the grid, financial incentives must outweigh the 
TABLE I

RESULTS FROM THE DEGRADATION COSTS AT THE END OF THREE-YEAR PERIOD.

\begin{tabular}{c|c|c}
\hline Degradation cost & Constant & Dynamic \\
\hline \hline Revenue $(\$)$ & 1880.9 & 2477.6 \\
Total V2G energy (MWh) & 372.3 & 381.6 \\
V2G energy per EV (MWh) & 7.23 to 27.37 & 19.07 to 19.09 \\
EV battery capacity $(\%)$ & 67.8 to 75.6 & 71.02 to 71.03 \\
\hline
\end{tabular}

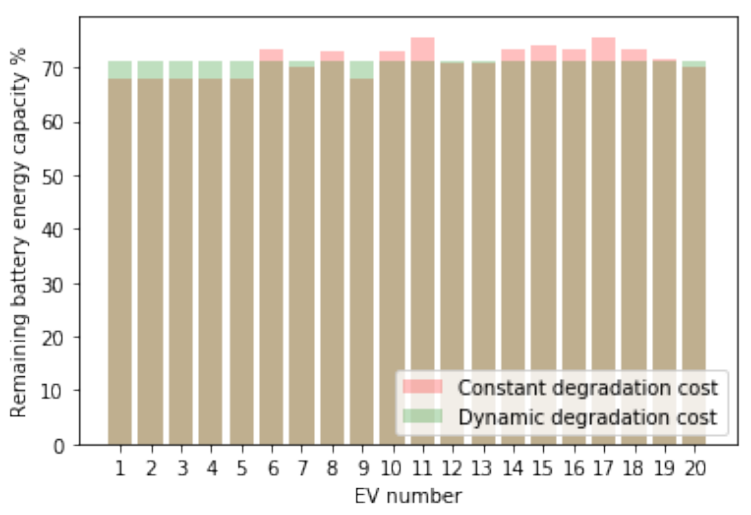

Fig. 2. Bar chart highlighting the remaining battery energy capacity in the EVs utilized for V2G using the constant and dynamic degradation costs.

losses due to degradation that support the grid event. The simulations are run for just one V2G application - energy arbitrage and just twenty EVs that charge at a single CSO facility. This can be extended to multiple EVs and optimizing for multiple V2G applications governed by Aggregators that work with various CSO facilities. Also, the battery model used is an empirical model, different battery models with thermal models can be tried and tested with blockchains to realize the full extent of possibility.

\section{CONCLUSiOnS}

A blockchain network is developed with the intent to assess and track the degradation cost in EV batteries. The consortium blockchain consists of all the actors involved in maximizing the utilization of a parked EV like the EV user, EV CSO, Aggregator, and EV MSP. The blockchain will log data that is important in accurately estimating the EV degradation cost and also includes data about the cash flow among the various actors for $\mathrm{V} 2 \mathrm{G}$ applications. Blockchains are more secure than regular databases, and consensus mechanisms make it harder to actively hack into the ledger. Modifying past transactions after they have been added to the blockchain is impossible. With worries about cybersecurity issues for EV charging networks, blockchains can help secure information transfer among various entities.

The results of using the dynamic degradation cost tracked by the blockchain in a smart charging MILP were compared to the results using a constant degradation cost in the same MILP. In a simulated scenario with a single EV CSO that used 20 Nissan Leaf EVs for energy arbitrage for a three-year period, it was seen that the dynamic degradation cost resulted in the prioritization of EVs with higher remaining battery life percentages for V2G. Meanwhile, the constant degradation cost picked EVs at random for $\mathrm{V} 2 \mathrm{G}$. The range of final EV battery capacities and the range of V2G energy per EV was a thousand times smaller for the dynamic degradation cost case when compared to the constant degradation cost case.

The data log also has other useful applications. Based on a week's data of an EV and data from other EVs of the same EV model, it will be possible to predict the useful life of the EV's battery. With this information, the EV user will have more power in making decisions whether partaking in V2G or not. At the end of the battery's life, i.e, when the battery loses 30\% of initial capacity, the records of the EV battery can be utilized for a second-life battery tracking for other grid applications. This method of tracking the battery's life can be implemented for battery swap applications as well.

\section{REFERENCES}

[1] S. Han, S. Han, and K. Sezaki, "Development of an optimal vehicle-togrid aggregator for frequency regulation," IEEE Transactions on smart grid, vol. 1, no. 1, pp. 65-72, 2010.

[2] E. Sortomme and M. A. El-Sharkawi, "Optimal scheduling of vehicle-togrid energy and ancillary services," IEEE Transactions on Smart Grid, vol. 3, no. 1, pp. 351-359, 2011.

[3] H. Farzin, M. Fotuhi-Firuzabad, and M. Moeini-Aghtaie, "A practical scheme to involve degradation cost of lithium-ion batteries in vehicle-togrid applications," ieee transactions on sustainable energy, vol. 7, no. 4, pp. 1730-1738, 2016.

[4] K. Chaudhari, A. Ukil, K. N. Kumar, U. Manandhar, and S. K. Kollimalla, "Hybrid optimization for economic deployment of ess in pv-integrated ev charging stations," IEEE Transactions on Industrial Informatics, vol. 14, no. 1, pp. 106-116, 2017.

[5] S. Nakamoto, "Bitcoin: A peer-to-peer electronic cash system," Manubot, Tech. Rep., 2019.

[6] C. Chen, J. Wang, F. Qiu, and D. Zhao, "Resilient distribution system by microgrids formation after natural disasters," IEEE Transactions on smart grid, vol. 7, no. 2, pp. 958-966, 2015.

[7] S. Hua, E. Zhou, B. Pi, J. Sun, Y. Nomura, and H. Kurihara, "Apply blockchain technology to electric vehicle battery refueling," in Proceedings of the 51st Hawaii International Conference on System Sciences, 2018.

[8] K. Christidis and M. Devetsikiotis, "Blockchains and smart contracts for the internet of things," Ieee Access, vol. 4, pp. 2292-2303, 2016.

[9] Y. Xiong, B. Wang, C.-c. Chu, and R. Gadh, "Vehicle grid integration for demand response with mixture user model and decentralized optimization," Applied energy, vol. 231, pp. 481-493, 2018.

[10] H. Liu, Z. Hu, Y. Song, and J. Lin, "Decentralized vehicle-to-grid control for primary frequency regulation considering charging demands," IEEE Transactions on Power Systems, vol. 28, no. 3, pp. 3480-3489, 2013.

[11] D. B. Richardson, "Electric vehicles and the electric grid: A review of modeling approaches, impacts, and renewable energy integration," Renewable and Sustainable Energy Reviews, vol. 19, pp. 247-254, 2013.

[12] S. N. Gowda, T. Zhang, C. J. Kim, R. Gadh, and H. Nazaripouya, "Transmission, distribution deferral and congestion relief services by electric vehicles," in 2019 IEEE Power \& Energy Society Innovative Smart Grid Technologies Conference (ISGT). IEEE, 2019, pp. 1-5.

[13] S. Pelletier, O. Jabali, G. Laporte, and M. Veneroni, "Battery degradation and behaviour for electric vehicles: Review and numerical analyses of several models," Transportation Research Part B: Methodological, vol. 103, pp. 158-187, 2017.

[14] J. Wang, J. Purewal, P. Liu, J. Hicks-Garner, S. Soukazian, E. Sherman, A. Sorenson, L. Vu, H. Tataria, and M. W. Verbrugge, "Degradation of lithium ion batteries employing graphite negatives and nickel-cobaltmanganese oxide+ spinel manganese oxide positives: Part 1, aging mechanisms and life estimation," Journal of Power Sources, vol. 269, pp. 937-948, 2014.

[15] D. Wang, J. Coignard, T. Zeng, C. Zhang, and S. Saxena, "Quantifying electric vehicle battery degradation from driving vs. vehicle-to-grid services," Journal of Power Sources, vol. 332, pp. 193-203, 2016. 